\title{
Theoretical Study on Bonding Nature of Rare Gases with Iron
}

\author{
DiHao Tan ${ }^{1}$ and An Yong $\mathrm{Li}^{2}$ \\ ${ }^{1}$ Southwest University \\ ${ }^{2}$ SouthWest University
}

May 5, 2020

\begin{abstract}
Chemical bonding nature of rare gases with various elements has been attracting permanent attention of chemical researchers. Interactions between transition metals and rare gases have special characters and should be an important subject of research. Here we theoretically studied the interacting properties of rare gas atoms with iron by inspecting the diatomic molecules FeRg $\left(\mathrm{Rg}=\mathrm{He}^{\sim} \mathrm{Rn}\right)$ and the Rg-inserting compounds $\mathrm{FeRgF}^{-/+}$and $\mathrm{HRgFe}^{-/+}(\mathrm{Rg}=\mathrm{Kr}, \mathrm{Xe}, \mathrm{Rn})$. Quantum chemistry methods DFT (B3LYP), MP2 and MP4(SDQ) with the basis sets aug-cc-pVTZ were employed to calculate the structure, frequencies and the thermodynamic properties of a series of the dissociation processes. These Rg-containing compounds are found to be thermodynamically stable. The NBO and AIM analyses and the electron localization function (ELF) were employed to study the bonding nature of rare gases with iron. These compounds are linear structures, the Laplacian distribution of electron density and ELF distribution are not axial symmetry at the part of Fe. The electron density distribution does not always show a BCP between Rg and Fe. These phenomena are a result of the incompletely filled $3 d$ shell of Fe. There are many new special characters of chemical bonding between $\mathrm{Rg}$ and transition metals.
\end{abstract}

\section{Hosted file}

Theoretical Study on Bonding Nature of Rare Gases with Iron.doc available at https: //authorea.com/users/301282/articles/431092-theoretical-study-on-bonding-nature-of-raregases-with-iron 